\title{
Penn ar Roz, un assemblage lithique de la transition Pléistocène-Holocène à Châteaulin (Finistère)
}

Penn ar Roz, a lithic assemblage of the Pleistocene-Holocene transition in Châteaulin (Finistère)

Penn ar Roz, ein litisches Fundensemble vom Übergang Pleistozän-Holozän aus Châteaulin (Finistère, Bretagne, Frankreich)

Stéphan Hinguant, Nicolas Naudinot, Miguel Biard, Alain Hénaff et Éric Nicolas

\section{OpenEdition}

Journals

Édition électronique

URL : http://journals.openedition.org/rao/3247

DOI : 10.4000/rao.3247

ISSN : 1775-3732

Éditeur

Presses universitaires de Rennes

Édition imprimée

Date de publication : 31 décembre 2016

Pagination : 21-37

ISBN : 978-2-7535-5683-6

ISSN : 0767-709X

\section{Référence électronique}

Stéphan Hinguant, Nicolas Naudinot, Miguel Biard, Alain Hénaff et Éric Nicolas, « Penn ar Roz, un assemblage lithique de la transition Pléistocène-Holocène à Châteaulin (Finistère) », Revue archéologique de l'Ouest [En ligne], 33 | 2016, mis en ligne le 31 juillet 2019, consulté le 02 mars 2021. URL : http://journals.openedition.org/rao/3247 ; DOI : https://doi.org/10.4000/rao.3247 


\title{
Penn ar Roz, un assemblage lithique de la transition Pléistocène-Holocène à Châteaulin (Finistère)
}

\author{
Penn ar Roz, a Lithic Assemblage of the Pleistocene-Holocene Transition \\ in Châteaulin (Finistère)
}

\author{
Stéphan Hinguant ${ }^{\mathrm{a}}$, Nicolas Naudinot ${ }^{\mathrm{b}}$, Miguel Biard ${ }^{\mathrm{c}}$, Alain HÉnAfF ${ }^{\mathrm{d}}$ \\ et Éric NicOlas ${ }^{\mathrm{e}}$
}

\begin{abstract}
Résumé : Menée sur une superficie de 34000 mètres carrés, sur un rebord de plateau dominant un méandre de l'Aulne, une fouille préventive sur le site de Penn ar Roz à Châteaulin (Finistère) a livré une quantité importante de données archéologiques couvrant une large chronologie. Parmi celles-ci, la mise au jour d'un assemblage lithique attribuable à une occupation post-azilienne documente ainsi la préhistoire, dans un secteur géographique peu étudié jusqu’ici.

Malgré un contexte pour partie remanié et la dispersion spatiale de la collection, des pièces caractéristiques (armatures, grattoirs sur lame...), mais surtout la constance dans le mode de débitage (production à la pierre tendre de lames régulières au profil rectiligne et de section plate à partir de deux plans de frappe opposés), sont en effet des éléments suffisamment diagnostiques pour éclairer la question de l'attribution chronoculturelle. Le site de Penn ar Roz alimente ainsi un peu plus la connaissance de cette période charnière entre Pléistocène et Holocène, aujourd'hui au cœur d'une importante dynamique de recherche dans le Grand Ouest de la France, en nous renseignant sur l'occupation de l'extrémité occidentale de la péninsule armoricaine par ces derniers groupes paléolithiques.
\end{abstract}

\begin{abstract}
A CRM excavation of the Penn ar Roz site in Châteaulin in Finistère (Brittany, France) by INRAP allowed the discovery of various archaeological records attributed to a wide chronology. A lithic assemblage attributed to the Pleistocene-Holocene transition was identified among Iron Age and medieval artifacts collected on this $34,000 \mathrm{~m}^{2}$ site located on a plateau above a large meander of the Aulne River. The assemblage is technologically homogeneous despite an important dispersal of the artifacts and obvious taphonomic issues. The various technical characteristic highlighted by our study suggest this assemblage to be the result of an installation of post-azilian communities at the very end of the Upper Paleolithic durind the GS1 (Younger Dryas)-Holocene transition. This discovery adds new evidence for the understanding of this key period in Western France. It brings new information about the situation of the western extremity of the Armorican peninsula during this period and asks the question of adaptation of these very demanding lithic productions in a raw materials scarcity context.
\end{abstract}

Mots clés : Finistère, Paléolithique supérieur final, industrie lithique, Transition Pléistocène-Holocène.

Keywords: Finistère, Final Upper Paleolithic, Lithic industry, Pleistocene-Holocene transition.

\footnotetext{
a INRAP-Bretagne, 37 rue du Bignon, 35577 Cesson-Sévigné et UMR 6566 du CNRS, CReAAH, Campus de Beaulieu, Université de Rennes 1, 35042 RENNES cedex. (stephan.hinguant@inrap.fr)

b Université Côte-d'Azur, CNRS, UMR 7264 CEPAM. (nicolas.naudinot@cepam.cnrs.fr)

"INRAP-Haute-Normandie, immeuble Mermoz, 30 boulevard de Verdun, 76120 Le Grand-Quevilly et UMR 7041 du CNRS "ArScAn", Nanterre. (miguel.biard@inrap.fr)

d Laboratoire LETG-Brest Géomer, UMR 6554 du CNRS, Institut Universitaire Européen de la Mer, rue Dumont-D'Urville, 29280 PLOUZANÉ. (alain. henaff@univ-brest.fr)

'INRAP-Bretagne, 37 rue du Bignon, 35577 CESSON-SÉVIGNÉ. (eric.nicolas@inrap.fr)
} 


\section{INTRODUCTION}

Entre Azilien et Mésolithique, à la charnière entre les derniers temps du Tardiglaciaire et les premiers moments de l'Holocène, autour de 9500 cal. BC, se développent des sociétés aux productions lithiques originales et en rupture avec les périodes qui les encadrent. Connus de la péninsule ibérique au sud de la Scandinavie, ces sites peuvent être regroupés au sein d'un grand techno-complexe composé de différentes traditions régionales (Laborien, Laborien récent, Ahrensbourgien, Épiarhrensbourgien). Ce grand ensemble est caractérisé par des productions laminaires élaborées, structurées autour de la recherche de lames régulières de section aplatie (FBT - Flat Blades and Bladelets Techno-complex; Naudinot et Jacquier, 2014). Les recherches sur ces dernières sociétés paléolithiques se sont multipliées depuis les années 1990 (voir par ex. Valentin, 1995; Fagnart, 1997) mais cette période est cependant restée longtemps méconnue dans le Grand Ouest de la France où les rares assemblages étudiés jusqu'au début des années 2000 étaient considérés comme magdaléniens ou "épimagdaléniens ». La reprise de collections anciennes, comme celle du site du Camp d'Auvours (Saint-Mars-laBrière, Sarthe; Naudinot, 2008, 2010), mais également la mise en place de nouvelles opérations programmées, comme à la Fosse (Villiers-Charlemagne, Mayenne; Naudinot et Jacquier, 2014), ont néanmoins permis de pallier ce retard. Cette dynamique a permis de proposer non seulement une nouvelle organisation chronoculturelle des sites tardiglaciaires et du début de l'Holocène dans la région (Marchand et al., 2004; Naudinot, 2008, 2010, 2013), mais également de commencer à aborder les systèmes paléo-économiques de ces groupes (Naudinot et Jacquier, 2014; Jacquier et Naudinot, 2015). La plupart des gisements mobilisés dans ces travaux sont toutefois localisés sur les marges orientales du Massif armoricain. Jusqu'à la découverte du gisement de Penn ar Roz dans le cadre de l'archéologie préventive, et hormis quelques indices souvent isolés (Marchand et al., 2004), les sites attribuables à la transition Pléistocène-Holocène restaient inexistants à l'extrémité de la péninsule armoricaine. Cette découverte permet ainsi d'étendre l'enquête sur ces groupes dans l'Ouest de la France, notamment en permettant de développer une réflexion sur les stratégies de gestion mises en place par ces sociétés aux débitages lithiques exigeant, dans un contexte géologique contraignant ne fournissant que de petits galets de silex de qualité hétérogène issus de cordons littoraux.

\section{DÉCOUVERTE ET PRÉSENTATION DU CORPUS}

Un projet d'aménagement d'une ZAC couvrant une surface de vingt hectares au nord-est de la ville de Châteaulin a conduit à la réalisation d'un diagnostic puis d'une fouille archéologique par l'un de nous (Nicolas, 2013) (fig. 1).

La fouille, centrée sur la zone la plus dense en vestiges $\left(34000 \mathrm{~m}^{2}\right)$, a mis au jour des structures excavées et du mobilier archéologique protohistorique et historique témoignant d'une occupation longue mais discontinue du territoire. Si le diagnostic archéologique avait permis d'identifier une présence néolithique dans les environs immédiats de l'emprise, sous la forme de rares pièces lithiques (notamment dans une fosse isolée), aucun artefact ni aucune structure n'accrédite la période sur la zone décapée. L’assemblage dont il est question dans cet article témoigne en revanche d'une présence plus ancienne sur le site sous la forme d'une petite installation pouvant être rapportée à l'extrême fin du Tardiglaciaire.

La collection est constituée de 180 pièces lithiques provenant pour l'essentiel de la couche limoneuse apparaissant directement sous la terre végétale (tableau 1). Quelques pièces sont néanmoins issues des comblements de structures protohistoriques, en position secondaire. Il s'y ajoute huit pièces macrolithiques qui ne seront pas prises en compte dans cette étude, aucun stigmate d'utilisation ne permettant de les attribuer à une collection préhistorique. Leur présence sur le site ne peut cependant s'expliquer que par un apport anthropique. Il s'agit de galets marins de roches variées (trois en quartz, un en microgranite, un en grès quartzite), de deux galets fortement brûlés (un en grès et un en quartz)

\begin{tabular}{|l|c|c|}
\hline Type & $\mathrm{nb}$ & $\%$ \\
\hline Éclats & 67 & 37,2 \\
\hline Lames & 59 & 32,7 \\
\hline Tablettes & 15 & 8,3 \\
\hline Crêtes & 13 & 7,2 \\
\hline Nucléus & 12 & 6,7 \\
\hline Sous-crêtes & 5 & 2,7 \\
\hline Éclats laminaires & 3 & 1,7 \\
\hline Indéterminé & 3 & 1,7 \\
\hline Éclat de crête & 1 & 0,6 \\
\hline Chute & 1 & 0,6 \\
\hline Éclat de quartz (non taillé?) & 1 & 0,6 \\
\hline Total & 180 & 100 \\
\hline
\end{tabular}

Tableau 1 : Châteaulin, Penn ar Roz. Décompte général de l'assemblage lithique.

Table 1: Quantification of the lithic assemblage. 
Figure 1 : Localisation géographique du site de Penn-ar-Roz (C) Géoportail - IGN).

Figure 1: Geographical location of the Penn-ar-Roz site.

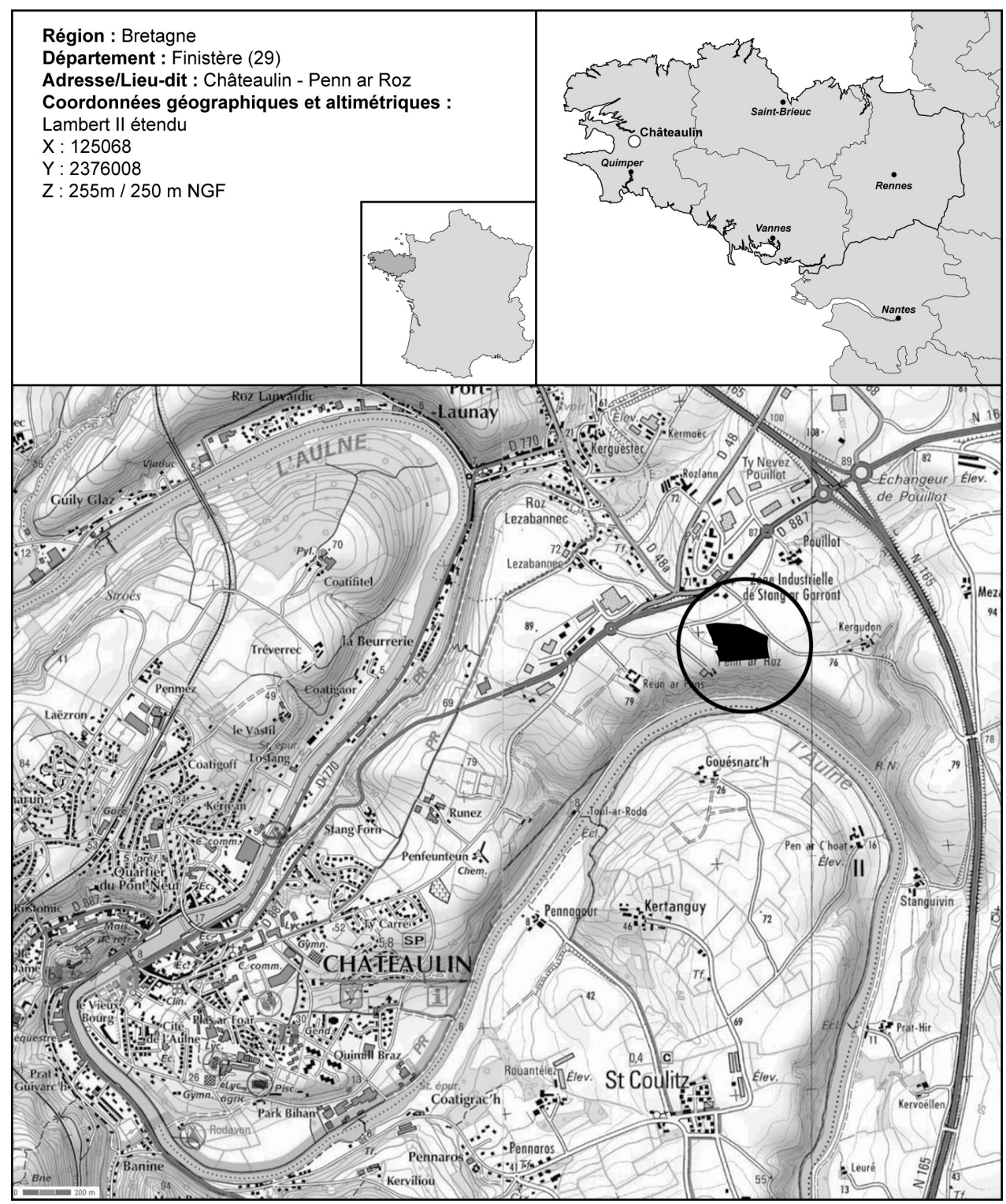

et enfin d'un galet allongé en roche volcano-sédimentaire, le seul portant des traces d'utilisation (surface lissée d'une des faces et encoche et écrasements sur l'un des bords) et correspondant à un probable lissoir d'attribution chronologique incertaine. L'absence de percuteur en lien avec la série lithique est donc patente. Signalons ici que deux nucléus, appartenant probablement à une composante mésolithique voire néolithique (boucharde?) sont également exclus de cet inventaire et que certains éclats, à défaut d'une diagnose suffisante, peuvent aussi poser la question de leur attribution chronoculturelle.

Ce corpus est donc très modeste et le contexte stratigraphique ainsi que la dispersion de l'ensemble invitaient à la prudence avant toute conclusion chronoculturelle. Il demeu- rait toutefois suffisamment important pour en proposer une étude techno-typologique et, sur un ensemble de critères qualitatifs, pour le rattacher plus précisément à la transition Pléistocène-Holocène, soit autour de 9700 cal. BC. Des assemblages régionaux contemporains, longtemps délaissés ou à tort attribués à un "Épimagdalénien ", sont aujourd'hui bien connus et participent à un large techno-complexe européen (Marchand et al., 2004; Naudinot, 2008, 2010, 2013).

\section{LE CONTEXTE NATUREL}

Le site de Penn ar Roz se localise sur la partie proximale de l'éperon topographique délimité par le méandre de l'Aulne 
à Châteaulin à l'aval du méandre de Saint-Coulitz (fig. 2). Il s'agit d'une partie d'un plateau culminant vers 95 mètres d'altitude NGF dans laquelle l'Aulne est encaissée de 75 à 80 mètres environ. Le site domine ainsi la vallée de l'Aulne vers le sud et le versant méridional de cette partie du plateau rejoint le fleuve par une forte pente correspondant à la rive concave d'un méandre. De courts talwegs orientés vers le sud incisent le plateau et le versant méridional, mais la pente générale du terrain est orientée vers le nord-ouest. On se trouve ici à la tête d'un talweg plus important et plus profondément incisé qui rejoint l'Aulne au nord, à la terminaison aval du méandre de Châteaulin au niveau de Port-Launay. Le site se trouve ainsi largement exposé au nord-ouest.

La surface du plateau est formée dans les roches sédimentaires du bassin de Châteaulin. Le substrat en affleurement correspond aux schistes et wackes dits de Pont-de-Buis, datés du Viséeen supérieur et du Namurien. Il s'agit d'une roche constituée d'une alternance de grès argileux et d'argilites dont certaines renferment localement des faunes et des flores fossiles. La schistosité est importante et l'aspect général est celui d'une ardoise qui a d'ailleurs été exploitée par des ardoisières.

Sous les formations superficielles, le substrat rocheux présente une grande fracturation de surface et montre des altérations plus ou moins poussées de la roche comportant des poches d'altération (parties gréseuses) entourées de bancs de schistes à veines quartzeuses largement fracturés, orientés d'une manière générale NNW-SSE, dans le sens de la plus grande pente. En première approche, cette fracturation de surface, qui concerne les premiers décimètres d'épaisseur de roche, résulterait localement de la cryoclastie qui s'ajoute à la fracturation naturelle de la roche à proximité de la surface (diaclase, décompression, retrait/gonflement des argiles).

Les formations superficielles qui recouvrent ce substrat rocheux sont peu épaisses, notamment dans la partie sommitale du site proche du versant méridional du plateau. La coupe réalisée selon la pente a permis de les observer sur une trentaine de mètres de longueur. Elles sont épaisses de moins d'un mètre et même plutôt de l'ordre de 0,80 mètre. Il s'agit de sédiments fins composés de limons compactés comportant quelques éléments grossiers de roches (cailloutis) épars (fig. 3). Des poches de graviers altérés forment des discontinuités dans cette formation limoneuse de surface et se présentent sous la forme de rubans obliques et en position singulière au sein des limons (fig. 4). L'examen de cette disposition morphologique particulière, qui se produit de manière plus ou moins nette tout au long de la coupe exami-

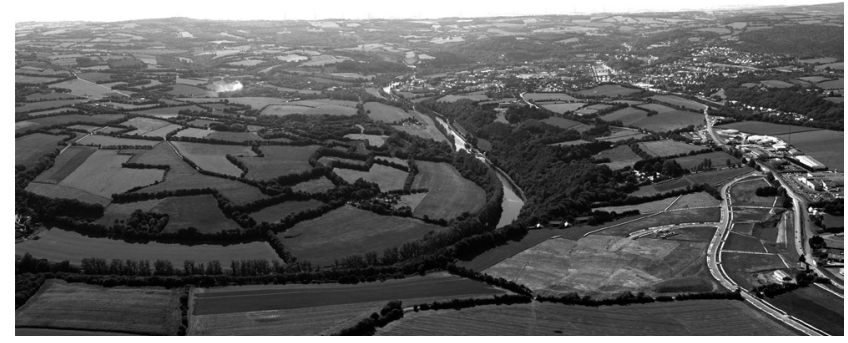

Figure 2 : Vue d'ensemble du site dans son contexte topographique. L'emprise du décapage archéologique est visible dans la partie inférieure droite du cliché () Hervé Paitier, Inrap).

Figure 2: Overview of the site in its topographical context. The excavation surface is visible in the lower right.

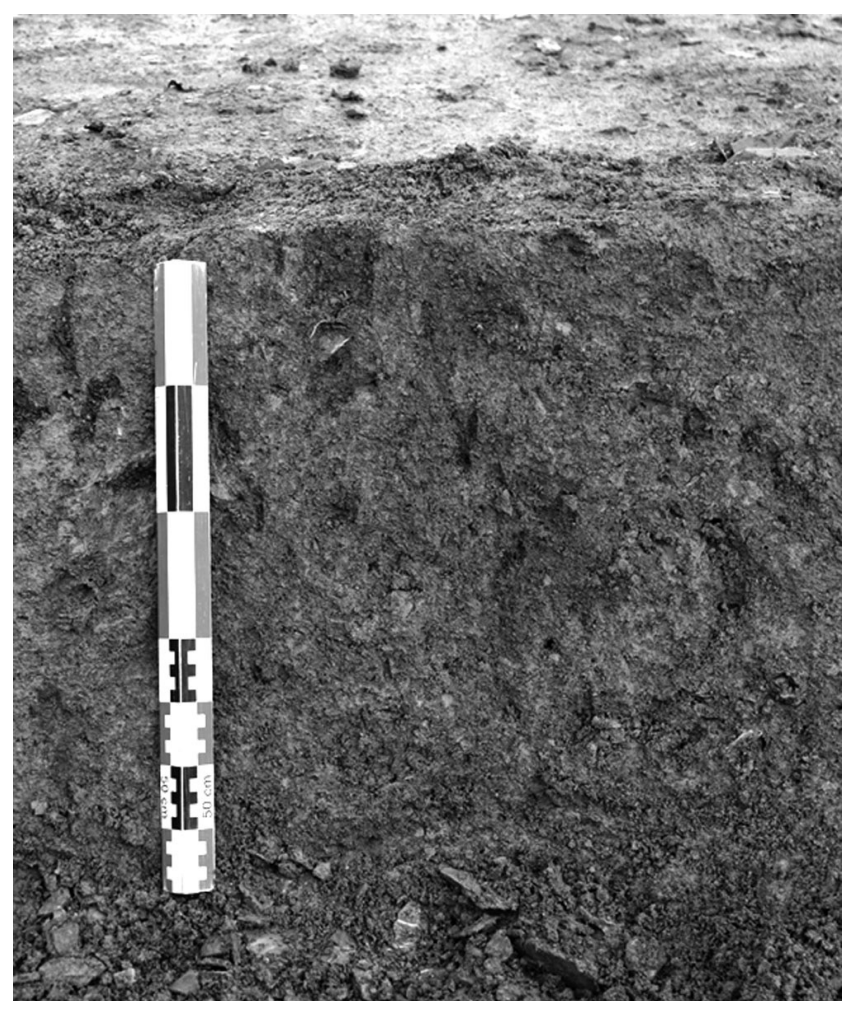

Figure 3 : Vue de détail des limons superficiels (C Éric Nicolas, Inrap).

Figure 3: Detailed view of superficial silts.

née, amène à l'interpréter comme le résultat d'une poussée exercée par les limons fluant lentement depuis le sommet du versant sur la pente. Il résulte de ces observations que les pièces lithiques contenues dans cet ensemble sédimentaire sont en position secondaire, largement dispersées sur la totalité de l'emprise (fig. 5). 


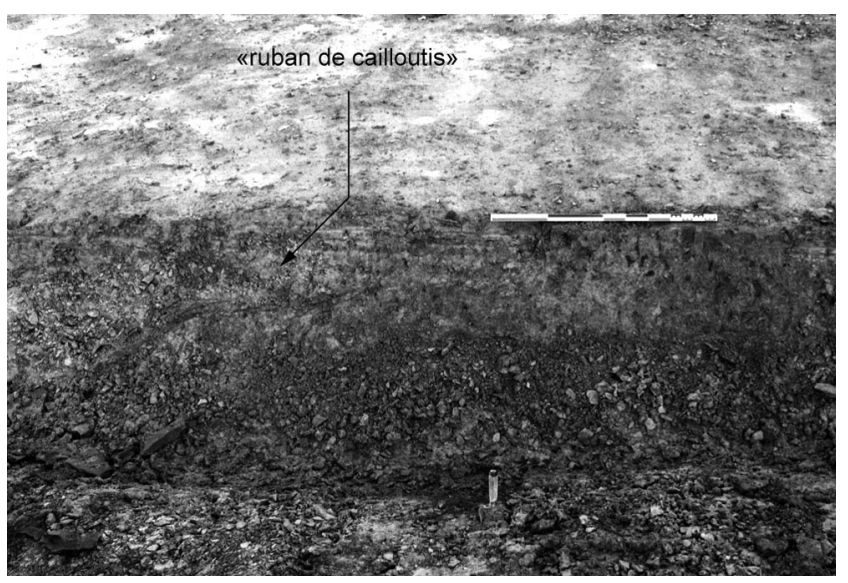

Figure 4 : Disposition singulière des poches de graviers altérés dans les limons résultant du fluage lent des formations superficielles le long de la pente du versant (C Éric Nicolas, Inrap).

Figure 4: Singular disposition of altered gravel pockets in silts resulting from the slow creep of sediments along the slope.

\section{L'ASSEMBLAGE LITHIQUE}

\section{État et homogénéité de la série}

La forte dispersion de la collection sur la surface étudiée et l'absence de toute concentration remarquable de pièces signent probablement une importante dégradation de l'état d'origine de l'occupation (fig. 5). Pour la majeure partie collectée à plus de $50 \mathrm{~cm}$ de profondeur, à l'interface des limons à cailloutis et des limons de surface, les pièces présentent pourtant peu de stigmates associés aux pratiques agricoles modernes, comme des traces de rouille sur les arêtes ou des cassures fraîches. On peut donc supposer un enfouissement ancien et rapide des pièces qui a favorisé leur préservation. Leur état est même très bon et les tranchants ne sont pas émoussés ni ne montrent d'esquillements ou d'ébréchures récents. Une patine affecte cependant de nombreuses pièces alors que d'autres ne présentent pas du tout cette altération. C'est sans doute à la nature colluviée et aux phénomènes de reptation des limons en lien avec la pente qu'il convient donc d'attribuer la répartition spatiale observée, sans que cela n'ait toutefois altéré considérablement l'état physique du mobilier. Dans le cadre de cette étude, une première recherche de raccords ou remontages n'a pas donné de résultats.

Le corpus compte seulement dix pièces brûlées ou présentant des traces de chauffe (faïençage, blanchiment, rubéfaction), l'une en position secondaire provenant d'une structure protohistorique. La répartition spatiale de ces pièces n'est pas significative et n'implique pas l'existence d'une ou plusieurs aires de chauffe.

Si plusieurs éléments suggèrent une certaine cohérence de la série, quelques pièces témoignent de la présence de composantes plus récentes et peuvent d'ores et déjà être

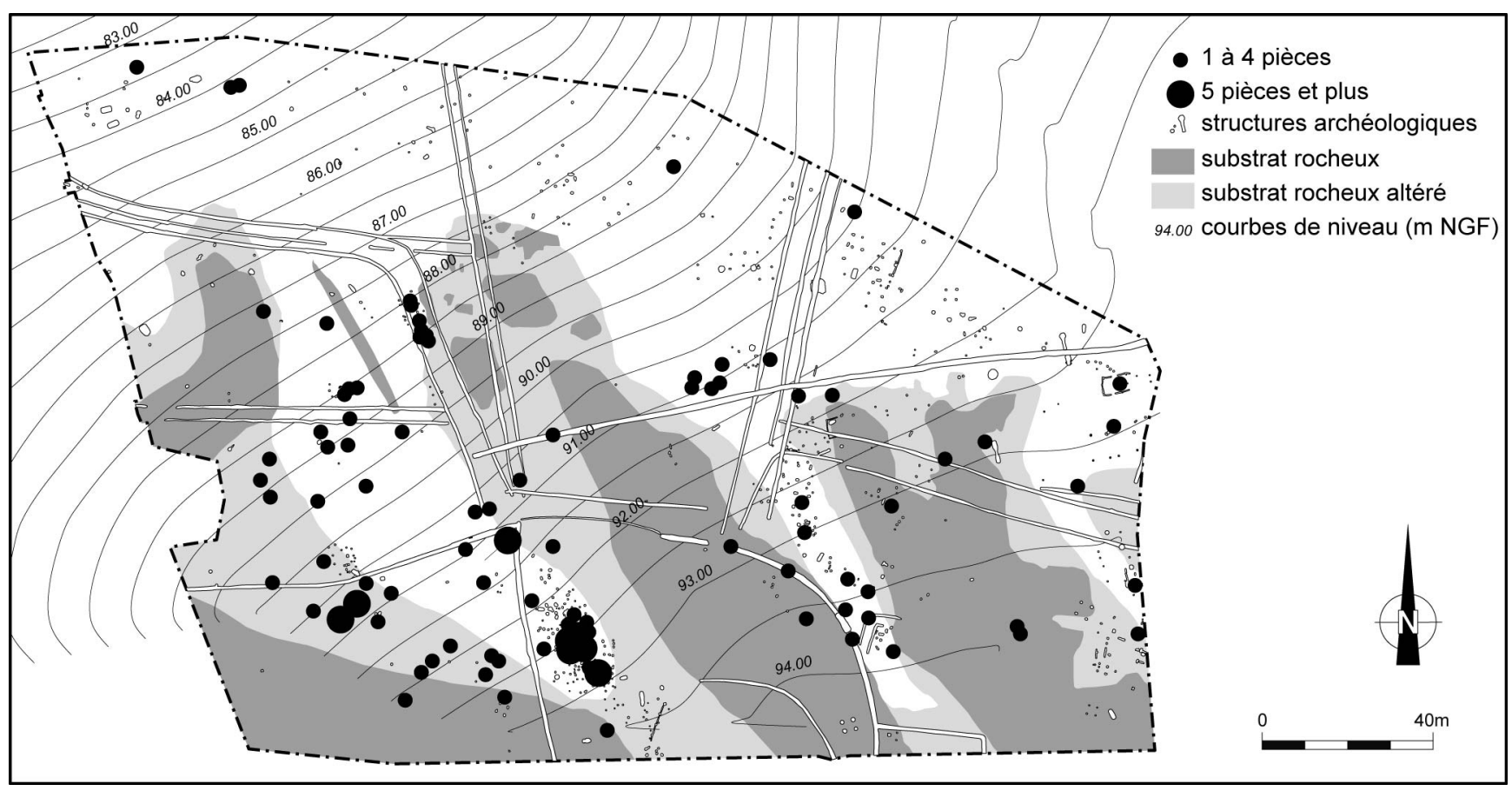

Figure 5: Répartition spatiale des pièces lithiques sur l'emprise de la fouille (C Éric Nicolas, Inrap).

Figure 5: Spatial distribution of lithic industry. 
écartées. Il s'agit en premier lieu de deux nucléus sur petits galets littoraux visant la production d'éclats allongés par le biais d'une exploitation unipolaire tournante pour l'un et multipolaire séquentielle pour l'autre. Une de ces pièces, découverte dans le comblement d'un fossé protohistorique au sud du site, pourrait d'ailleurs avoir été utilisée comme boucharde. En second lieu, la question de l'attribution chronologique d'un support laminaire bitronqué, probablement contemporain de l'occupation principale tardiglaciaire, pose toutefois, compte tenu du contexte, la question d'une attribution au Néolithique. Nous développerons le cas de cette pièce infra.

\section{Origine des ressources siliceuses exploitées}

Hormis un petit fragment de matériau schisteux dont l'attribution à la composante Tardiglaciaire n'est pas clairement démontrée, la quasi-totalité de l'assemblage a été débitée dans des matériaux siliceux. La très grande majorité des pièces sont en silex du Crétacé (95\%), matériau nécessairement allochtone dans ce secteur du Massif armoricain éloigné du cours de la Loire ou des cordons littoraux. Si l'origine littorale de ce silex gris à larges inclusions beiges/ blanches est attestée, la position précise des cordons exploités reste difficile à appréhender du fait de notre méconnaissance de la variabilité pétrographique de ces matériaux. Pour le moment, une collecte de galets de silex sur le littoral de la mer d'Iroise et plus précisément dans la baie de Douarnenez est privilégiée du fait de sa proximité avec le site ${ }^{1}$. Compte tenu des dimensions importantes des galets de silex débités ( $>10 \mathrm{~cm} / 15 \mathrm{~cm}<$ ), l'hypothèse d'un approvisionnement sur des cordons aujourd'hui submergés et situés à plus faible distance des affleurements du Crétacé supérieur est tout à fait envisageable. Ce scénario aurait à la fois pour mérite d'expliquer la rareté de volumes de gabarits similaires sur les actuels cordons littoraux finistériens ainsi que la diminution progressive des dimensions des galets récoltés au cours de la préhistoire. À ces silex littoraux s'ajoutent deux pièces retouchées (un grattoir sur lame à crête et une bitroncature trapézoïdale) fabriquées dans un silex noir à cortex alluvial rappelant les matériaux du Sénonien du Sud-Ouest du Bassin parisien. Récolté en position secondaire, il est impossible de préciser l'origine exacte de ce matériau même si l'axe ligérien, situé à environ trois cents kilomètres du site, est probable. Un grattoir sur crête à un pan a quant à lui été fabriqué dans un silex packstone gris/noir opaque détritique qui rappelle les matériaux de la bande jurassique encadrant la bordure orientale du Massif armoricain (silex bathoniens

1. Aujourd'hui au plus proche à une quinzaine de kilomètres du site de Penn ar Roz mais, compte tenu du trait de côte au moment du Dryas récent, probablement à plus de cinquante kilomètres lors de l'occupation. de la plaine de Caen ou bajociens du sud de la Sarthe). Du fait de la rareté de ces silex sur le site, ainsi que de la nature de ces pièces (toutes trois retouchées), leur introduction sous forme de tool kits est en tout cas attestée. Hormis ces silex, deux pièces témoignent de l'exploitation des grès lustrés régionaux, disponibles à une vingtaine de kilomètres au sud du site dans le secteur de Kervouster (Béchennec et al., 1999; Monnier, 1976) ou dans le fond de la baie de Douarnenez (Gouletquer et al., 1996).

\section{Étude typologique et technologique de la série}

L'assemblage lithique taillé de Penn ar Roz se caractérisant avant tout par sa dispersion sur l'ensemble de la surface fouillée, la synchronie de la collection n'est pas assurée de prime abord et nous n'avons qu'une vision tronquée de la chaîne opératoire mise en œuvre pour l'obtention des supports d'outils. Par analogie avec d'autres collections nous pouvons cependant établir le diagnostic techno-typologique de la série et tenter quelques comparaisons.

\section{L'outillage}

Loutillage retouché est mal représenté avec seulement huit pièces soit 4,5\% du corpus (tableau 2). Signalons ici que de nombreux produits lamino-lamellaires bruts (une trentaine de pièces dont dix entières) ont certainement été utilisés sur leurs tranchants ou extrémités pour diverses activités pour le moment inabordables en l'absence de véritable analyse fonctionnelle (fig. 6). À cet outillage a posteriori s'ajoute par ailleurs une pièce esquillée (non illustrée).

Les grattoirs sont majoritaires (5) et tous réalisés en bout de lames et lames à crête. Ils présentent des fronts semi-circulaires larges (fig. 7, $\mathrm{n}^{\text {os }} 1,2$ et 4 ) ou étroits (fig. $7, \mathrm{n}^{\text {os }} 3$ et 5) mais généralement circonscrits à l'extrémité du support. La retouche qui aménage ces fronts est plutôt convergente et régulière. Les supports choisis sont diversifiés puisque si des lames de bonne, voire très bonne, régularité ont été utilisées, trois grattoirs ont été fabriqués sur lames à crête, dont deux corticales (fig. $7, n^{\text {os }} 1$ et 3 ). On notera que deux de ces crêtes

\begin{tabular}{|l|c|}
\hline \multicolumn{1}{|c|}{ Type } & $\mathrm{nb}$ \\
\hline Armature & 2 \\
\hline Grattoir sur lame & 5 \\
\hline Lame retouchée & 1 \\
\hline Total & 8 \\
\hline
\end{tabular}

Tableau 2 : Châteaulin, Penn ar Roz. Décompte de l'outillage aménagé.

Table 2: Quantification of lithic retouched tool. 


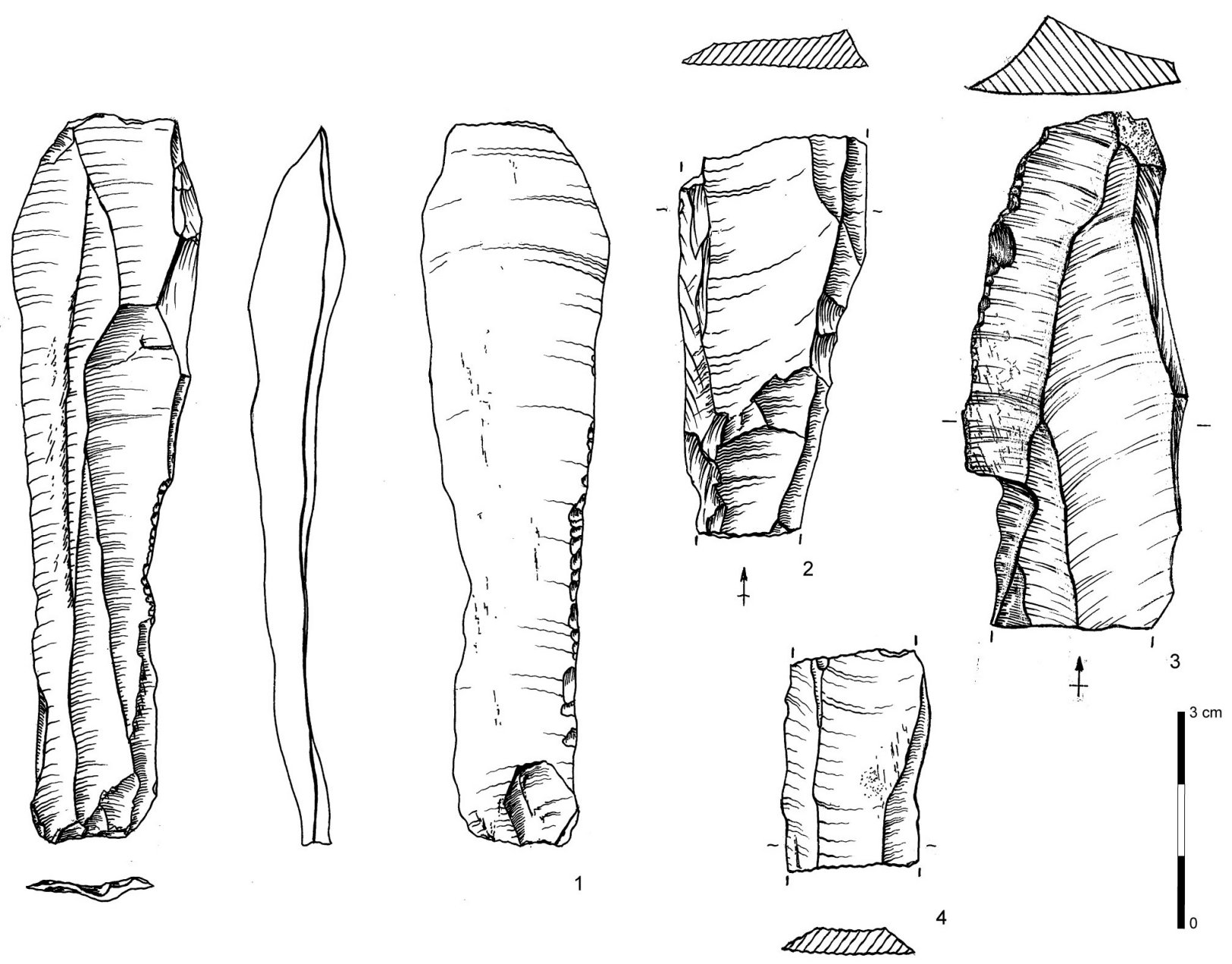

Figure 6 : Châteaulin, Penn ar Roz. Produits laminaires. 1 : lame retouchée; 2 : fragment proximo-mésial de lame sous-crête; 3 : mésiodistal de lame sous-crête utilisée; 4 : mésial de lame brute (C S. Hinguant, Inrap).

Figure 6: Blade industry. 1: retouched blade; 2: proximo-mesial fragment of sub-crested blade; 3: mesio-distal fragment of used sub-crested blade; 4: mesial fragment of unretouched blade.

sont débitées dans des matériaux allochtones (Sénonien et Bajocien-Bathonien). Les deux exemplaires cassés montrent une fracture en flexion sur leur face dorsale qui pourrait résulter de l'utilisation ou de la retouche de l'objet emmanché, ou bien d'une segmentation volontaire des supports pour la production de ces outils (fig. 7, $\mathrm{n}^{\text {os }} 4$ et 5).

Enfin, malgré quelques tamisages réalisés dans les zones de plus forte concentration, les éléments de pointes de projectile sont très rares dans l'assemblage. On note toutefois la présence d'un fragment apical de pointe à dos à troncature très oblique brisé lors de la phase de régularisation du dos (fig. $7, \mathrm{n}^{\circ} 7$ ). Bien que la fragmentation de cette pièce et sa singularité au sein de l'assemblage ne permette pas de conclure sur son attribution, sa morphologie tout comme les choix techniques mis en œuvre pour sa fabrication (notamment celui de l'extrémité proximale du support pour l'aménagement de l'apex et la latéralisation du dos sur le bord droit du support), pourraient rapprocher cet élément des pointes des Blanchères (Rozoy, 1978), éléments caractéristiques des sociétés de la transition Pléistocène-Holocène, globalement entre le Sud-Ouest de la France (Langlais et al., 2014) et le nord du Bassin parisien (Valentin, 1995). Ces éléments sont également particulièrement bien représentés dans le Grand Ouest (Marchand et al., 2004; Naudinot, 2008, 2010, 2013).

On en retrouve plusieurs milliers d'exemplaires sur certains sites, comme au Camp d'Auvours dans la Sarthe (Naudinot, 2010). Elles sont également présentes en très grandes quantités à la Fosse en Mayenne (Naudinot et Jacquier, 2009, 2013,2014 ) ou encore, dans une moindre mesure, au sein du locus 28704 d'Alizay en Normandie (Bemilli et al., 2014), sites bénéficiant d'excellentes conditions de préservation et dont le dernier a été daté entre $9960 \pm 40 \mathrm{BP}$ (Beta333640) et $10100 \pm 40 \mathrm{BP}$ (Beta-333638) soit entre 9800 

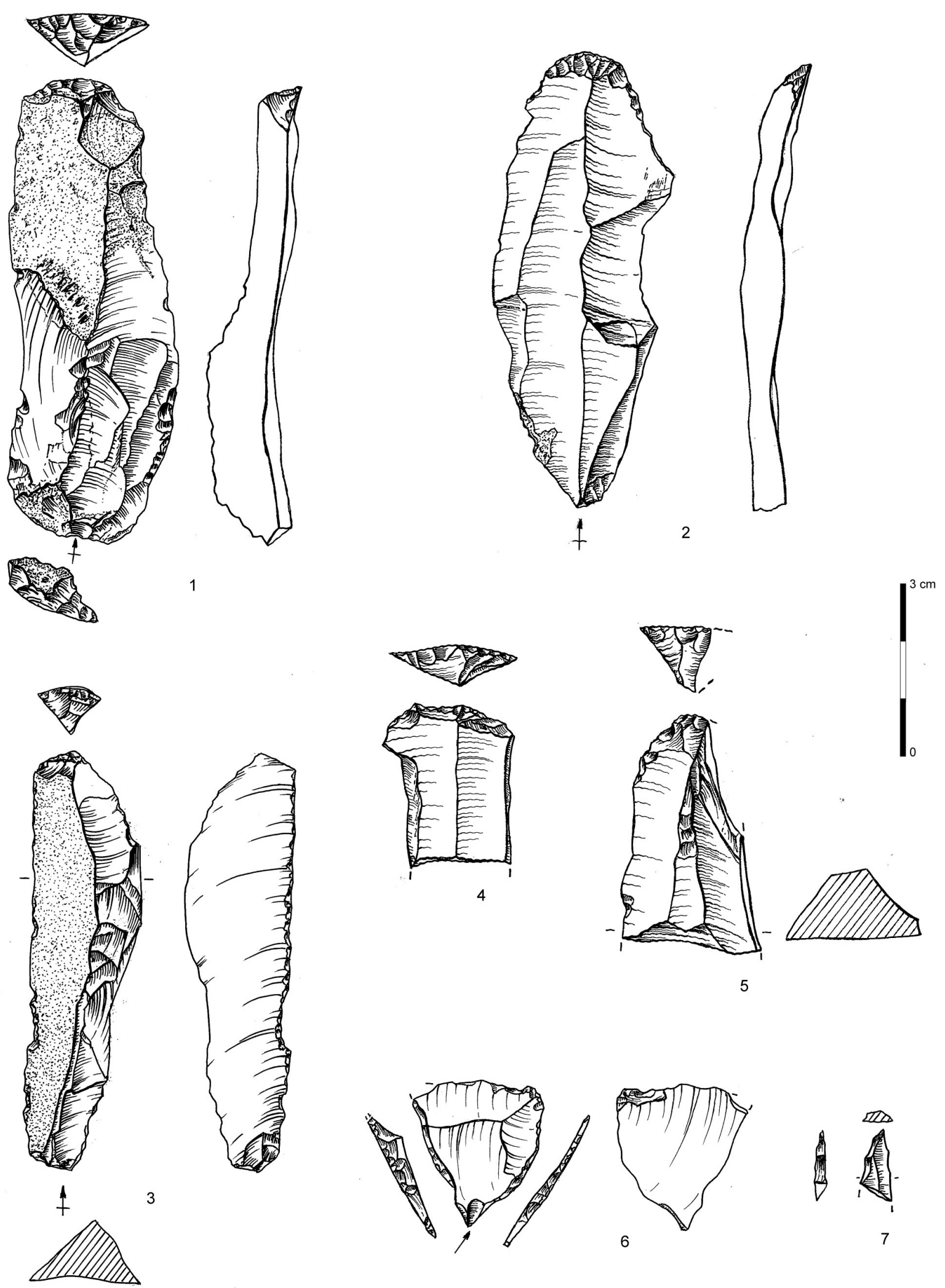

5
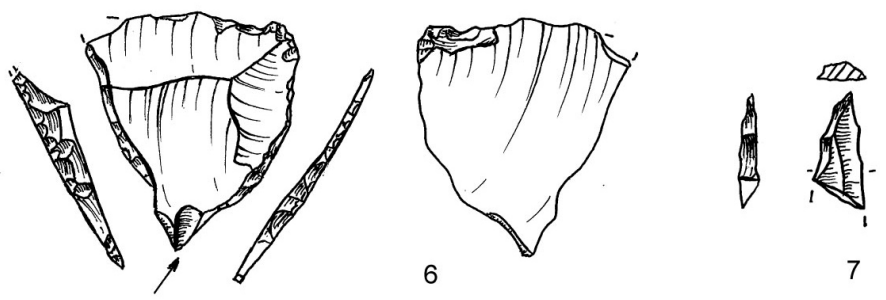

Figure 7 : Châteaulin, Penn ar Roz, industrie lithique, outillage retouché. 1 à 5 : grattoirs sur bout de lame; 6 : bitroncature trapézoïdale; 7 : apex de probable pointe des Blanchères (@ S. Hinguant, Inrap).

Figure 7: Retouched tools. 1 to 5: endscrapers; 6: trapezoid bitroncature; 7: possible "pointe des Blanchères" tip. 
et 9300 cal. BC. À cette pièce s'ajoute une bitroncature trapézoïdale que certaines caractéristiques rendent compatibles avec les pièces récemment mises en évidence pour la fin du Tardiglaciaire (Naudinot, 2008, 2010, 2013; fig. 7, n 6). Si les dimensions importantes de ce support s'inscrivent dans la marge haute de la variabilité morphométrique des bitroncatures tardiglaciaires, d'autres caractères - tels la régularité du support laminaire investi, sa section aplatie ou l'aspect vibré de sa face inférieure traduisant une extraction à la pierre tendre (Pelegrin, 2000; Biard et Prost, 2015), semblent tout à fait compatibles avec les exemplaires post-glaciaires. Cette bitroncature symétrique présente deux troncatures obliques directes abruptes (l'une droite en proximal et l'autre légèrement convexe en distal). La présence d'un enlèvement burinant de près de $17 \mathrm{~mm}$ de long sur la face supérieure, initié depuis sa grande base et associé à des ébréchures sur sa face inférieure, témoigne de l'usage de cette pièce comme armature à tranchant transversal. Ce mode d'emmanchement a également été récemment suggéré pour les exemplaires tardiglaciaires (Naudinot, 2013) notamment grâce au corpus de cinquante pièces de ce type découvert sur le site post-azilien de la Fosse à Villiers-Charlemagne (Naudinot et Jacquier, 2009, 2013, 2014). Si plusieurs éléments tendent donc à valider l'attribution de l'objet à la composante postazilienne de Penn ar Roz, sa singularité dans l'assemblage, ses dimensions importantes, tout comme la présence locale d'un Néolithique moyen et le contexte peu fiable du site, imposent cependant la plus grande prudence.

\section{Objectifs et méthode de production}

Si avec 67 pièces la catégorie des éclats domine l'assemblage (environ $37 \%$ ), la somme des produits laminolamellaires, sous crêtes et éclats allongés compte également 67 individus, représentant la même proportion dans la série (tableau 1). Les lames et les lamelles comptent 59 pièces, ce qui atteste de l'importance de l'objectif lamino-lamellaire. Cette prépondérance de l'intention lamino-lamellaire se confirme avec l'usage exclusif de produits allongés pour la conception de l'outillage, ainsi que par les dix nucléus attribués au Paléolithique supérieur final dont les derniers négatifs témoignent tous d'une exploitation des volumes dédiés à l'obtention de supports allongés. Sans être déterminant, ce constat chiffré est un premier argument à retenir pour qualifier l'homogénéité de la collection.

Les dimensions moyennes des lames sont de $15,6 \mathrm{~mm}$ (médiane = $14 \mathrm{~mm}$ ) de large pour une épaisseur de 4,3 $\mathrm{mm}$. La longueur moyenne des supports entiers est de 45,3 $\mathrm{mm}$ et une pièce atteint même $100 \mathrm{~mm}$. La production laminolamellaire vise essentiellement l'obtention de supports entre 9 et $20 \mathrm{~mm}$ de large (fig. 8). Ces lames sont plutôt régulières (indice $=2,1$ sur une échelle de $0-3$ ), rectilignes

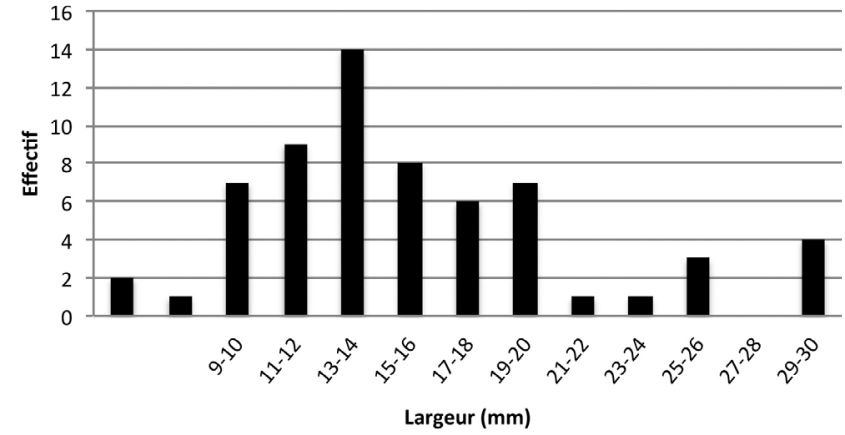

Figure 8 : Châteaulin, Penn ar Roz. Largeur moyenne des supports laminaires.

Figure 8: Average width of laminar blanks.

(indice $=2,5)$ et de section plate. Dans $75 \%$ des cas, elles sont a minima à trois pans. Si l'on écarte les deux nucléus sur petits galets probablement issus d'une composante plus récente, ces pièces lamino-lamellaires semblent donc bien correspondre aux objectifs identifiés sur le reste de l'assemblage. L'observation des derniers négatifs utiles sur les nucléus témoigne d'une largeur moyenne qui gravite autour de 14,5 mm (tableau 3).

Les grattoirs, tout comme la lame retouchée, ont été réalisés sur les supports les plus larges (23,4 $\mathrm{mm}$ de moyenne), mais pas les plus réguliers (indice $=1$ ). Pour les deux armatures, si la bitroncature a été confectionnée sur une lame large de $25 \mathrm{~mm}$, la pointe à dos, dont seul subsiste l'apex, semble avoir été réalisée sur un support plus étroit.

Le débitage semble avoir été mené d'un bout à l'autre de la chaîne opératoire à l'aide d'un percuteur minéral. Si les éclats de mise en forme ou d'entretien présentent des

\begin{tabular}{|l|c|c|}
\hline \multicolumn{1}{|c|}{$\mathrm{N}^{\circ}$} & largeurs & moy. \\
\hline 4140 & $5 / 8 /-$ & 6,5 \\
\hline $4148-1$ & $9 / 19 /-$ & 14 \\
\hline $4096-1$ & $17 /-$ & 17 \\
\hline 4147 & $19 /-$ & 19 \\
\hline 4111 & $15 /-$ & 15 \\
\hline 4067 & $9 /-$ & 9 \\
\hline 4073 & $18 /-$ & 18 \\
\hline $4139-1$ & $20 /-$ & 20 \\
\hline 4126 & $6 / 17 /-$ & 11,5 \\
\hline $4096-2$ & $14 / 15 /-$ & 14,5 \\
\hline Moyenne générale & & 14,5 \\
\hline
\end{tabular}

Tableau 3 : Châteaulin, Penn ar Roz. Largeurs des derniers enlèvements complets sur les nucléus lamino-lamellaires (en $\mathrm{mm}$ ). Table 3: Width of the last entire negative of removal on the cores. 
stigmates tout à fait compatibles avec une percussion à la pierre dure, les supports lamino-lamellaires, objectifs de la production à Penn ar Roz, suggèrent systématiquement l'usage d'un percuteur de pierre tendre, probablement un grès, dans un geste tangentiel. Il est d'ailleurs tout à fait possible que les éclats aient également été obtenus à l'aide du même outil de taille mais selon un geste différent, en percussion davantage rentrante. La plupart des supports présentent une préparation du bord du plan de frappe par abrasion même si elle semble toutefois moins poussée que sur d'autres séries régionales présumées contemporaines et l'analyse des nucléus montre d'ailleurs des bords de plan de frappe parfois denticulés.

Les volumes sont exploités à partir de deux plans de frappe opposés (tableau 4) selon une progression semi-tournante. La table est large et le débitage s'étend peu sur les flancs et jamais sur le dos. Cette stratégie pourrait signer une volonté d'obtention de supports rectilignes mais également étalés aux tranchants aigus, objectif qui pourrait être central dans les productions des groupes de l'extrême fin du Tardiglaciaire (Naudinot, 2013; Naudinot et Jaquier, 2013, 2014; Jacquier et Naudinot, 2015). Si le recours à deux plans de frappe est attesté par l'observation des nucléus à l'état d'abandon, les supports lamino-lamellaires présentant des négatifs d'enlèvements extraits à partir d'un plan de frappe opposé sont relativement rares au sein de la série (22\%). Ce pourcentage pourrait résulter soit d'une exploitation bipolaire dont les enlèvements se croisent assez peu sur la table, ce que pourrait suggérer le taux beaucoup plus important de négatifs bipolaires sur les extrémités distales $(54,5 \%)$ par rapport aux mésiales $(26 \%)$ ou proximales $(0 \%)$, soit plus vraisemblablement d'un rythme d'alternance variable entre les deux plans de frappe. Le deuxième plan de frappe semble dans tous les cas bien avoir eu une vocation productive, ce que montrent également les nucléus qui témoignent d'une exploitation bipolaire selon un rythme d'alternance plutôt rapide avant leur abandon (fig. 9, 10 et 11).

L'obtention des lames et lamelles recherchées est assurée par la mise en place d'une méthode de débitage soignée met-

\begin{tabular}{|l|c|c|}
\hline \multicolumn{1}{|c|}{$\mathrm{N}^{\circ}$} & Type de négatifs & $\mathrm{nb}$ \\
\hline 4140 & Multipolaire & 1 \\
\hline $\begin{array}{l}4096-1 / 4096-2 / 4139-1 / 4148- \\
1 / 4111 / 4147\end{array}$ & Bipolaire & 6 \\
\hline $4067 / 4126$ & Unipolaire & 2 \\
\hline 4073 & Unipolaire préférentiel & 1 \\
\hline Total & & 10 \\
\hline
\end{tabular}

Tableau 4 : Châteaulin, Penn ar Roz. Décompte des types de nucléus.

Table 4: Quantification of the types of cores. tant en jeu différents procédés de mise en forme et d'entretien. L'usage de crête, et plus particulièrement de crêtes latérales, est récurrent (fig. $7, \mathrm{n}^{\text {os }} 1,3$ et 5). Ce procédé a été mis en place durant toute la durée de l'exploitation laminolamellaire comme en témoigne une néo-crête sur lamelle. L'usage régulier de ce procédé reflète un soin important dans l'entretien des convexités des volumes exploités. Il est d'ailleurs intéressant de remarquer que, comme dans d'autres séries présumées contemporaines de la région (Naudinot, 2010), des lames sous-crêtes présentent des témoignages de la mise en place d'une nouvelle crête directement après leur extraction. Ce soin exacerbé mais obligatoire à l'usage d'une percussion tendre minérale est également perceptible dans les modalités d'entretien des angulations des plans de frappe. Avec quinze exemplaires, ces produits d'entretien sont très bien représentés dans l'assemblage (fig. 12). Une des tablettes étudiées a d'ailleurs été extraite sans qu'aucun support n'ait été débité depuis l'extraction de la tablette précédente. Ce soin est d'autant plus intéressant que le site se situe à une certaine distance des matériaux lithiques et que les volumes disponibles dans ces cordons littoraux ont des dimensions souvent peu importantes. On notera également que six des tablettes de l'assemblage ont été extraites depuis le flanc du nucléus (fig. 12, n 3 ). Ces tablettes " obliques" sont particulièrement récurrentes dans les assemblages contemporains de l'Ouest de la France (Naudinot, 2010, 2013) et semblent être destinées à réinitialiser le débitage sur les flancs. En amont de cette opération, il a en effet été nécessaire de déplacer l'axe du débitage de la table vers un des flancs, afin de bénéficier de bonnes angulations pour le détachement de crêtes ou de lames de flanc.

Les nucléus sont abandonnés très tôt (cf. fig. 9-11). Si sur trois d'entre eux la présence de réfléchissements, parfois nombreux, a pu contraindre à l'abandon (fig. 11, nº 2), sur quatre pièces, c'est plutôt l'aplatissement excessif de la table (voire la concavité pour une pièce; fig. $10, \mathrm{n}^{\circ} 1$ ) qui a motivé l'abandon. Ce phénomène, tout comme l'apparition de réfléchissements sur la table, est certainement le résultat d'une exploitation cherchant à produire des lames plates et rectilignes sur des surfaces aux faibles convexités. Pour trois autres pièces, l'abandon des volumes semble ainsi avoir été motivé par les objectifs de production et non pour des raisons techniques. Il semble donc bien que les tailleurs de Penn ar Roz aient essentiellement recherché des supports dont les largeurs moyennes étaient supérieures à $14 \mathrm{~mm}$ (cf. fig. 8 et tableau 3). Ce comportement est particulièrement intéressant, surtout si nous le replaçons dans le contexte d'une certaine pénurie de matériaux. Il est d'ailleurs rare dans l'Ouest de la France où, pour les périodes plus récentes, les nucléus sont généralement exploités jusqu’à exhaustion. Sur les sites régionaux contemporains, la production de lamelles, parfois 
Figure 9 : Châteaulin, Penn ar Roz. Nucléus bipolaire

(C) S. Hinguant, Inrap).

Figure 9: Two opposed platforms core.
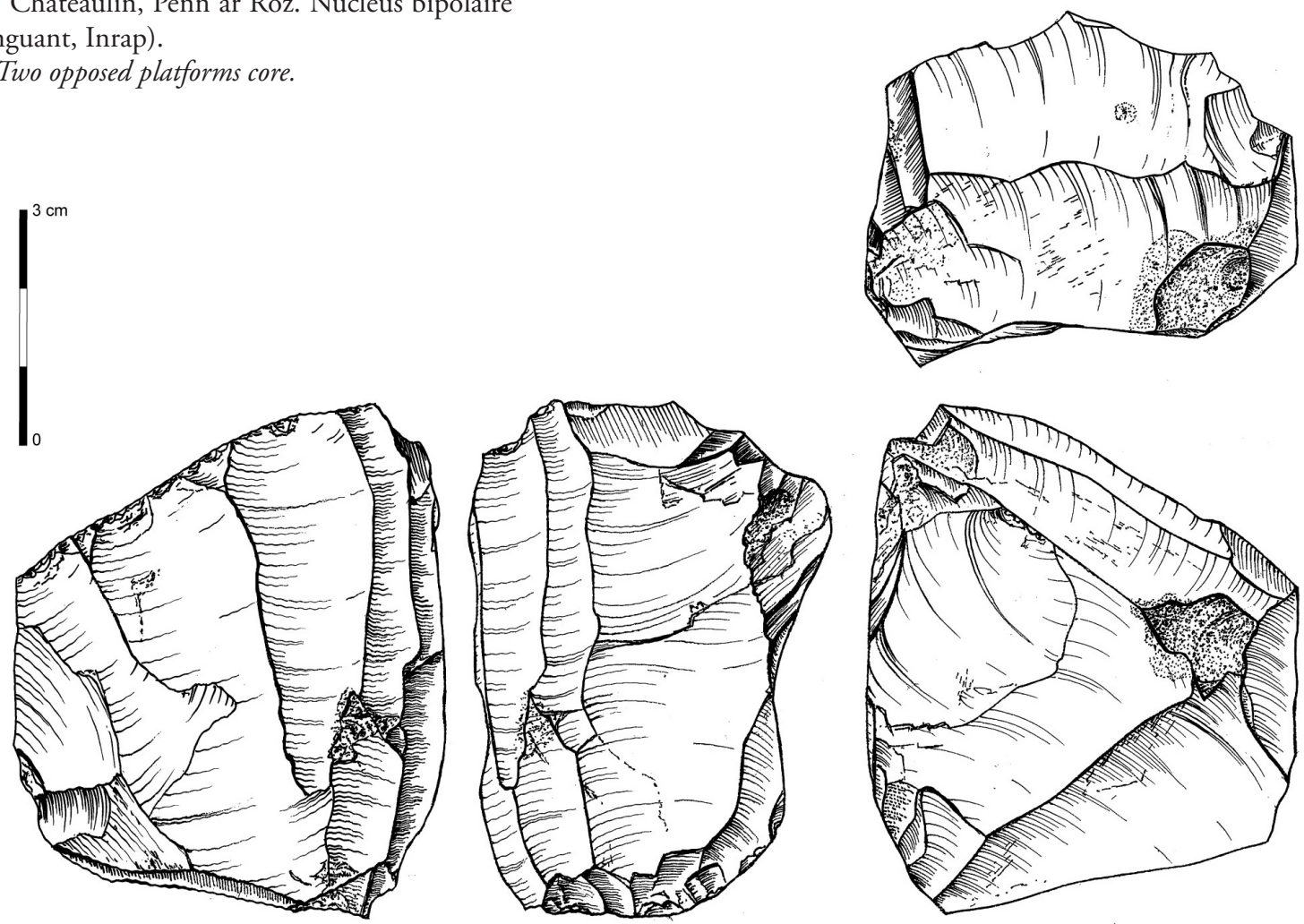

assez étroites, est presque toujours intégrée à l'exploitation laminaire des volumes (Naudinot, 2013). Ce comportement traduit un objectif de production assez strict, probablement en lien avec le statut du site mais malheureusement inabordable du fait des problèmes taphonomiques évoqués plus haut. On notera toutefois que deux des nucléus étudiés pourraient résulter d'une reprise postérieure du débitage sur des pièces abandonnées.

\section{UN NOUVEAU JALON OUEST ARMORICAIN POUR LES DERNIERS TEMPS du PaléOlithiQUe SUPÉRIEUR?}

Malgré le contexte en grande partie remanié et la dispersion spatiale de la collection, l'apparente homogénéité du lot suggère une certaine unicité et des pièces caractéristiques comme la constance dans le mode de débitage, sont suffisamment diagnostiques pour éclairer la question de l'attribution chronoculturelle. Le débitage soigné, l'importance de l'objectif lamino-lamellaire, la moindre représentativité de l'outillage sur éclat, tout comme la grande homogénéité des matériaux mis en œuvre sont ainsi autant de caractéristiques communes que l'on rencontre dans les séries tardiglaciaires même si une production similaire est connue dès le
Paléolithique supérieur ancien et jusqu'aux périodes récentes de la préhistoire. C'est dans la gestion soignée des volumes exploités, malgré la moindre qualité des galets de silex, que la distinction semble s'affirmer. La recherche de lames et lamelles très régulières, selon un mode de débitage bipolaire à deux plans de frappe opposés, caractérise la production. Les négatifs des derniers enlèvements comme la proportion de lames brutes et/ou retouchées donnent ainsi à la série une indéniable cohésion.

C'est avec les deux armatures, dont la bitroncature, que la question chronoculturelle s'affine vers l'extrême fin du Tardiglaciaire. Aucune pièce microlithique de la famille des géométriques n'est comptabilisée et certains aspects de l'armature perçante pourraient évoquer les éléments identifiés en contexte paléolithique. La question de la bitroncature, probable armature de flèche tranchante, a par ailleurs été récemment soulevée pour des séries attribuées au Paléolithique supérieur final (Naudinot, 2008, 2010, 2013; Naudinot et Jacquier, 2009). Des découvertes récentes (la Fosse en Mayenne, Alizay-Igoville dans l'Eure), mais aussi le réexamen de collections anciennes, suggèrent indéniablement que ce type d'objet apparaît bien à l'extrême fin du Tardiglaciaire (Naudinot, 2008, 2010, 2013; Aubry et al., 2011; Langlais et al., 2014). Le même constat a d'ailleurs été récemment avancé pour les pointes à troncature oblique 

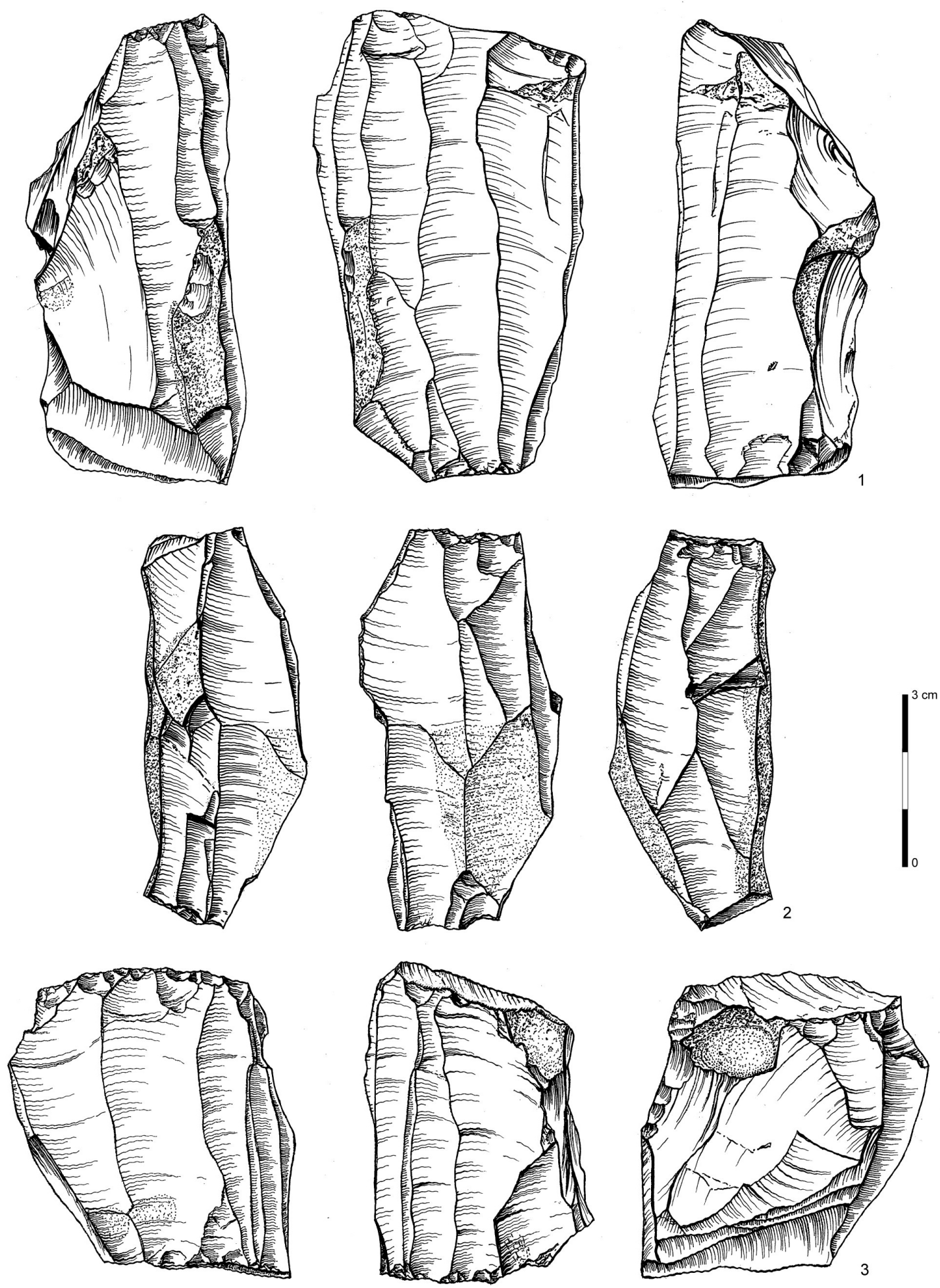

Figure 10 : Châteaulin, Penn ar Roz. Nucléus bipolaires (@ S. Hinguant, Inrap). Figure 10: Two opposed platforms cores. 

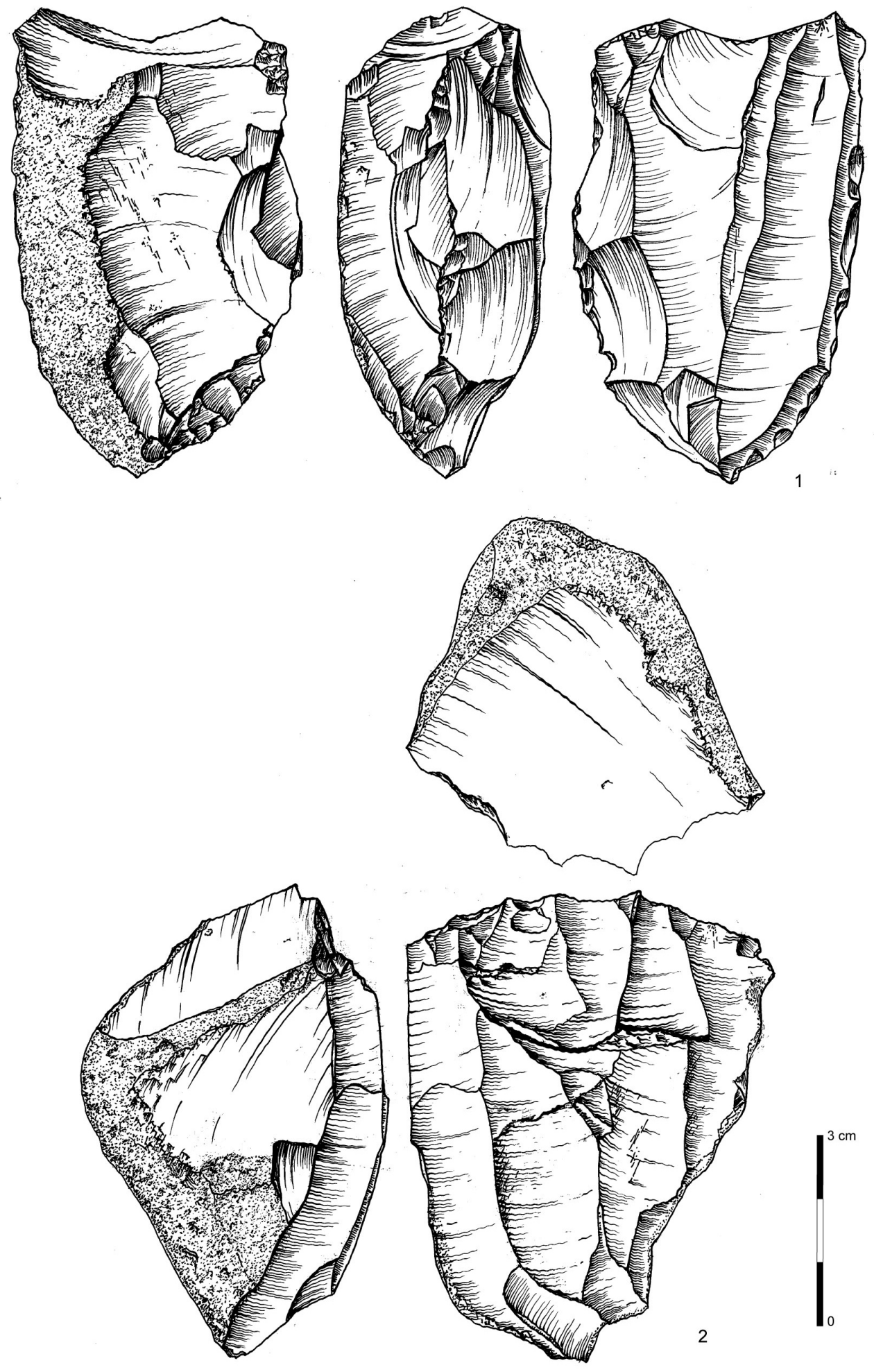

Figure 11 : Châteaulin, Penn ar Roz. Nucléus unipolaire $\left(\mathrm{n}^{\circ} 2\right)$ et unipolaire préférentiel ( $\left.\mathrm{n}^{\circ} 1\right)$ (@ S. Hinguant, Inrap). Figure 11: Unipolar core (N.2) and preferential unipolar core. 

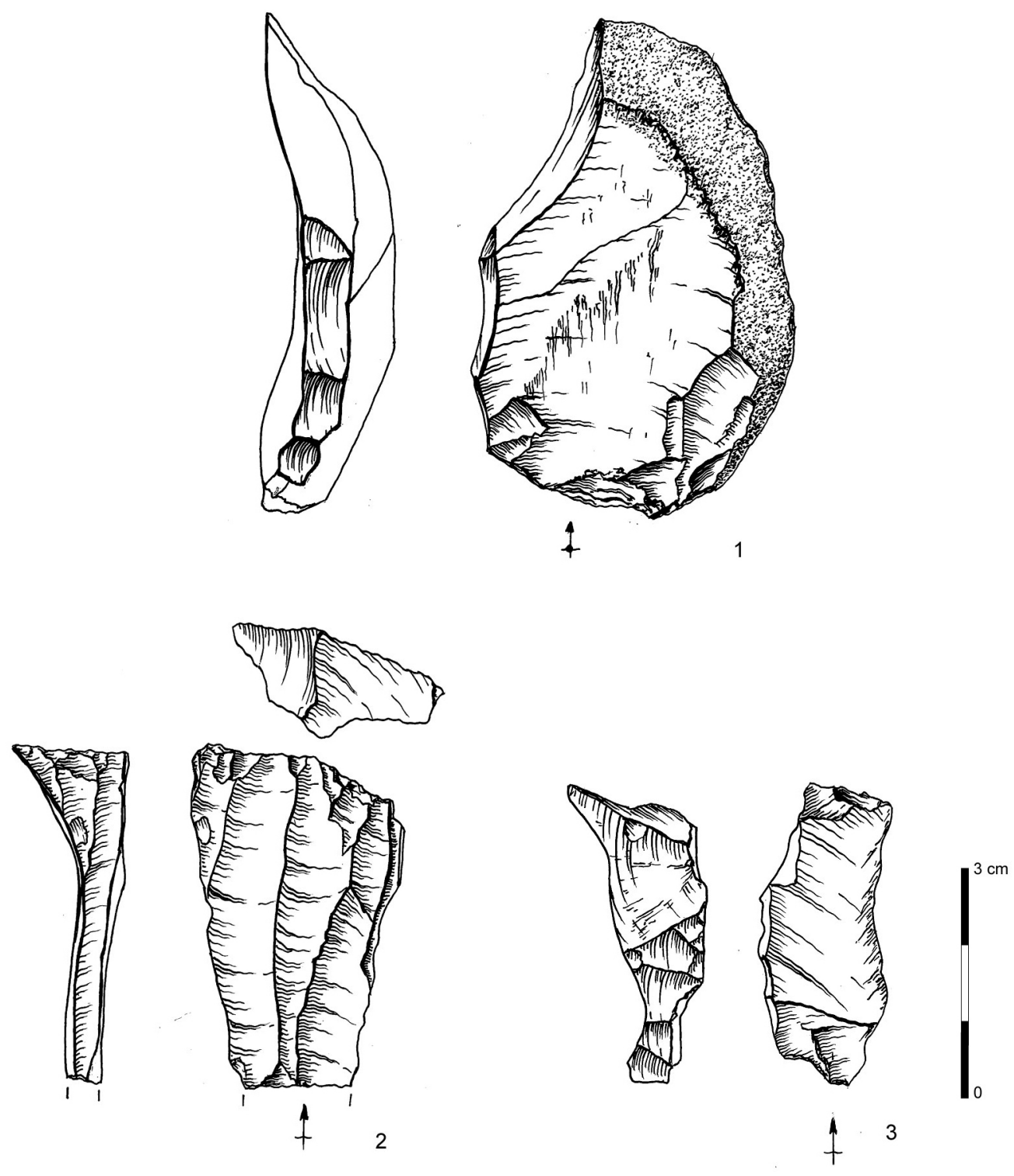

Figure 12 : Châteaulin, Penn ar Roz. Pièces techniques. 1 et 3 : tablettes; 2 : lame de flanc outrepassée (C S. Hinguant, Inrap). Figure 12: Technical pieces. 1 and 3: core tablets; 2: overpassed flank blade.

concave dont la mise en évidence sur des sites contemporains de la transition Pléistocène-Holocène va dans le sens d'une production antérieure au Mésolithique s.l. (Biard et Hinguant, 2004, 2011). L'association d'une production de lames régulières pour la fabrication d'un outillage domestique et de lamelles dédiées à celles d'armatures de traits, au cours d'une même chaîne opératoire, est ainsi un type d'assemblage lithique en passe de caractériser cette transition entre la fin des temps glaciaires et le début de l'Holocène. Si ce critère d'unicité technologique n'est pas fondamentalement prouvé au sein de la collection de Penn ar Roz, les similitudes observées avec les séries du Nord de la France et des régions limitrophes (Fagnart, 2009; Valentin, 2008;
Langlais et al., 2014), font que cette attribution chronologique pour le site breton n'aurait rien d'incongrue. On regrette à ce titre encore une fois le contexte stratigraphique peu fiable du site qui ne permet pas de trancher. La tendance qui se dessine demeure cependant bien celle d'une occupation post-azilienne, pouvant se placer dans la seconde moitié de l'intervalle 10 500-9000 cal. BC.

Enfin, par sa position topographique dominant un large méandre de l'Aulne, le site rappelle la fréquence du choix de ce type d'implantation par les groupes tardiglaciaires post-aziliens dans l'Ouest de la France (Naudinot, 2010). Plus que la position de hauteur, c'est davantage la proximité du cours d'eau et surtout la boucle formée par la rivière 
qui semble avoir été privilégiée. L'inventaire des sites de la période dans l'Ouest de la France met d'ailleurs en avant ces choix, qui peuvent s'expliquer par l'éventuelle concentration de ressources saisonnières, peut-être halieutiques (Naudinot, 2010, p. 278), comme sur le modèle des sites de la Fosse (Mayenne) ou de la Cadiais (Ille-et-Vilaine).

Du fait de son contexte peu assuré et de la taille réduite de son corpus lithique, le site de Penn ar Roz ne constitue bien évidemment pas un gisement majeur pour la caractérisation des derniers temps du Tardiglaciaire en Europe occidentale. Par l'homogénéité de son assemblage et sa position à l'extrémité de la péninsule armoricaine, il permet toutefois de contribuer à la récente dynamique de recherche mise en place ces dernières années dans la région pour la connaissance du Tardiglaciaire. Cette étude apporte notamment des éléments de réflexions intéressants sur les stratégies de gestion des matériaux lithiques par ces groupes de la transition. Contrairement à la plupart des gisements présumés contemporains de la région, essentiellement localisés dans la moitié orientale du Massif armoricain, Penn ar Roz est en effet situé beaucoup plus loin des grands affleurements de silex des bassins sédimentaires encadrant ce massif cristallin. L'analyse présentée montre que la rareté et les dimensions réduites des rognons de silex disponibles dans les cordons littoraux les plus proches du site, n'ont pas conduit à une réduction des exigences qualitatives des productions. Cette " pénurie » en volumes de bonne qualité ne semble d'ailleurs pas avoir affecté non plus la productivité du débitage comme en témoignent les nucléus régulièrement abandonnés dans les phases laminaires du débitage, sans exploitation lamellaire ou même production expédiente d'éclats en fin de chaîne opératoire, comportements probablement à mettre en relation avec les activités pratiquées sur le site de Penn ar Roz. Les résultats obtenus sur cet assemblage, lorsque mis en parallèle avec les données récentes acquises ces dernières années (Valentin, 2008; Bodu et al., 2011; Naudinot et Jacquier, 2014), devraient toutefois permettre de contribuer à la délicate question de l'organisation socio-économique de ces groupes de la transition Pléistocène-Holocène.

\section{Bibliographie}

Aubry B., Thomann A., Biard M., Bemilli C., Santiago-Lara V., Tessier V. et Honoré D., 2011 - «Une occupation du Tardiglaciaire; Alizay-Igovillle (Eure) ", Journées archéologiques de Haute-Normandie, Harfleur, 23-25 avril 2010, p. 9-24.

Béchennec F., Hallégouët B. et Thiéblemont D. avec la collaboration de Guerrot C., Cocherie A. et Carn A., 1999 - Notice explicative. Carte géologique de la France (1/50 000), feuille Quimper (346), Orléans, BRGM, 161 p. Carte géologique par F. BÉchennec et B. Hallégouët (1999).

Bemilli C., Biard M., Chaussé C. et Donnart K., 2014 - «Une partie de chasse à l'Aurochs, il y a 10000 ans. Le locus 28704 d'Alizay (Eure, France) ", in Costamagno S. (dir.), Histoire de l'alimentation humaine : entre choix et contraintes. Actes du 138e congrès des sociétés historiques et scientifiques, Rennes, 22-27 avril 2013, p. 170-187.

Biard M. et Hinguant S., 2004 - « Paléolithique supérieur final ou Mésolithique ancien ? Le site du Buhot à Calleville (Eure) ", Bulletin de la Société préhistorique française, 101, 3, p. 597-600.

Biard M. et Hinguant S. (dir.), avec les contributions de Beurion C., Deloze V., Forré P. et Sellami F., 2011 - Le bivouac préhistorique du Buhot à Calleville (Eure) : caractérisation techno-typologique d'un assemblage lithique lamino-lamellaire de la fin du Paléolithique supérieur, Paris, CNRS/Inrap, coll. "Recherches archéologiques ", 280 p.

Biard M. et Prost D., 2015 - "Le débitage à la pierre tendre : exemple de deux postes de taille de l'extrême fin du Paléolithique en Haute-Normandie ", Bulletin de la Société préhistorique française, 112, 1, p. 59-73.

Bodu P., Olive M., Valentin B., Bignon-Lau O. et Debout G., 2011 - «Où sont les haltes de chasse ? Discussion à partir des sites tardiglaciaires du Bassin parisien ", in Bon F., Costamagno S. et Valdeyron N. (dir.), Haltes de chasse en Préhistoire. Quelles réalités archéologiques?, Actes du colloque international, université Toulouse II - Le Mirail, 13-15 mai 2009, P@lethnologie, 3, p. 231-252.

FAGNART J.-P., 1997 - La fin des temps glaciaires dans le Nord de la France. Approche archéologique et environnementale des occupations humaines du Tardiglaciaire, Paris, Société préhistorique française, Mémoire 24, 270 p.

FAGNART J.-P., 2009 - « Les industries à grandes lames et éléments mâchurés du Paléolithique final du Nord de la France : une spécialisation fonctionnelle des sites Épi-ahrensbourgiens ", in Crombé P., Van Strydonck M., Sergant J., Boudin M. et Bats M. (éd.), Chronology and Evolution within the Mesolithic of North-West Europe. Proceedings of an International Meeting, Brussels, 30 mai - 1er juin 2007, Newcastle, Cambridge Scholars Publishing, p. 39-55.

Gouletquer P.-L., Kayser O., Le Goffic M., Léopold P., Marchand G. et Moullec J.-M., 1996 - « Où sont passés les Mésolithiques côtiers Bretons ? Bilan 1985-1995 des prospections de surface dans le Finistère ", Revue archéologique de l'Ouest, 13, p. 5-30.

Jacquier J. et Naudinot N., 2015 - "Recycling, maintaining and anticipating: the various significance of management strategies at the end of the Pleistocene in Northwestern France”, Quaternary International, 361, 10, p. 269-287

Langlais M., Detrain L., Ferrie J.-G., Boudadi-Maligne M., Mallye J.-B., Marquebielle B., Rigaud S., Bonnet- 
Jacquement P., Fat Cheung C., Naudinot N., Valdeyron N. et Turq A., 2014 - «Réévaluation d'une série éponyme et perspectives pour le Laborien du Sud-Ouest ", in Langlais M., Naudinot N. et Peresani M., (éd.), Les sociétés de l'Allerød et du Dryas récent entre Atlantique et Méditerranée. Actes de la séance de la Société préhistorique française (Bordeaux, 24-25 mai 2012), Paris, Société préhistorique française, p. 83-128.

Marchand G., Blanchet S., Chevalier G., Gallais J.-Y., Le Goffic M., Naudinot N. et Yven E., 2004 - « La fin du Tardiglaciaire sur le massif armoricain : territoires et cultures matérielles ", Paleo, 16, p. 137-170.

Monnier J.-L., 1976 - «Le gisement moustérien de tradition acheuléenne de Kervouster en Guengat (Finistère). Fouilles de 1974, 1975 et 1976 ", Bulletin de la Société archéologique du Finistère, 104, p. 15-26.

Naudinot N., 2008 - "Les armatures lithiques tardiglaciaires dans l'Ouest de la France (régions Bretagne et Pays de la Loire) : proposition d'organisation chrono-culturelle et chaîne opératoire de fabrication ", in Pétillon J.-M., DiasMeirinho M.-H., Cattelain P., Honegger M., Normand C. et Valdeyron N. (coord.), Recherches sur les armatures de projectiles du Paléolithique supérieur au Néolithique. Actes du colloque C83, XVe congrès de l'UISPP, Lisbonne, 4-9 septembre 2006, P@lethnologie, 1, p. 250-277.

Naudinot N., 2010 - Dynamiques techno-économiques et de peuplement au Tardiglaciaire dans le Grand-Ouest de la France, thèse de doctorat de l'université de Rennes 1, 731 p.

Naudinot N., 2013 - «La fin du Tardiglaciaire dans le Grand Ouest de la France ", Bulletin de la Société préhistorique française, 110, 2, p. 233-255.

Naudinot N. et JACQUier J., 2009 - « Un site tardiglaciaire en place à la Fosse (Villiers-Charlemagne, Mayenne) : premiers résultats et implications chrono-culturelles ", Bulletin de la Société préhistorique française, 106, 1, p. 145-158.
Naudinot N. et Jacquier J., 2013 - Le site Paléolithique final de la Fosse (Mayenne). Approche palethnologique d'une occupation de la transition Pléistocène-Holocène sur les bords de la Mayenne, Rapport final d'opération programmée (triennale 2009-2011), Service régional d'archéologie des Pays de la Loire, 252 p.

Naudinot N. et JACQuier J., 2014 - « Socio-economic organization of Final Paleolithic societies: New perspectives from an aggregation site in Western France ", Journal of Anthropological Archaeology, 35, p. 177-189.

Nicolas É. (dir.), avec la collaboration de Blanchet A., Brisotto V., Cherel A.-F., Daoulas G., Guitton V., Hénaff A., Hinguant S., Jouanet N., Labaune-Jean F., Le Forestier $S$. et Seignac H., 2013 - Penn ar Roz (Châteaulin, Finistère), un site d'activité métallurgique protohistorique et antique, Rapport final d'opération, Inrap Grand-Ouest, 327 p.

Pelegrin J., 2000 - «Les techniques de débitage laminaire au Tardiglaciaire : critères de diagnose et quelques réflexions ", in Valentin B., Bodu P. et Christensen M. (dir.), L'Europe centrale et septentrionale au Tardiglaciaire. Actes de la table ronde internationale de Nemours, 13-16 mai 1997, Nemours, APRAIF, Mémoires du musée de Préhistoire d'Île-de-France, 7, p. 73-86.

Rozoy J.-G., 1978 - Les derniers chasseurs. L'épipaléolithique en France et en Belgique. Essai de synthèse, Bulletin de la Société archéologique champenoise, 3 vol.

VAlentin B., 1995 - Les groupes humains et leurs traditions au Tardiglaciaire dans le Bassin parisien. Apports de la technologie lithique comparée, Thèse de doctorat de l'université de Paris 1 , 3 vol.

VALENTIN B., 2008 - Jalons pour une paléohistoire des derniers chasseurs (XIVe-VIe millénaire avant J.-C.), Paris, Publications de la Sorbonne, Cahiers archéologiques de Paris 1, 1, 325 p. 
Zusammenfassung: Penn ar Roz, ein litisches Fundensemble vom Übergang Pleistozän-Holozän aus Châteaulin (Finistère, Bretagne, Frankreich) - Eine Notgrabung des Institut national des recherches archéologiques préventives (INRAP), die auf einer Fläche von etwa $34000 m^{2}$ im Bereich der Fundstelle von Penn ar Roz bei Châteaulin (Finistère, Bretagne, Frankreich) stattfand, hat zahlreiche archäologische Hinterlassenschaften zu Tage gefördert, die verschiedene Siedlungsaktivitäten über einen weiten chronologischen Zeitraum belegen. Die Fundstelle befindet sich auf einem Plateau, das eine Flussschleife des Flusses Aulne überragt. Neben eisenzeitlichen und mittelalterlichen Fundobjekten befindet sich unter den Funden auch ein lithisches Fundensemble, das einer einer post-azilienzeitlichen Besiedlung vom Übergang Pleistozän (Jüngere Dryas, GS-I)-Holozän in einem bisher selten untersuchten Gebiet zugewiesen werden kann. Trotz einiger taphonomischer Probleme und einer räumlichen Verteilung der Funde, bei der von einer teilweisen Verlagerung der Funde ausgegangen werden muss, ist das Fundensemble als einheitlich zu betrachten. Dies gilt für seine signifikanten Fundstücke (Pfeilbewehrungen, Klingenkratzer...), jedoch insbesondere für die verwendete Schlagtechnik (Geräte aus weichem Gestein, u.a. regelmäßige Klingen mit geradem Profil, die im Querschnitt flach sind und zwei sich gegenüberliegende Schlagflächen aufweisen). Dabei handelt es sich um Charakteristika, die hinreichend spezifisch sind, um die vorgenommene chronologische Einordnung zu bestätigen. Die Fundstelle von Penn ar Roz bereichert unsere Kenntnis dieser wichtigen Übergangsperiode zwischen Pleistozän und Holozän, die heute im Zentrum der im westlichen Frankreich vorherrschenden Forschungsinteressen steht. Sie belegt eine Besiedlung am westlichen Rand der armorikanischen Halbinsel durch die letzten paläolithischen Gruppen.

Schlüsselwörter: Finistère, Endpaläolithikum, Lithische Industrie, Pleistozän-Holozä. 